\title{
Diagnostic and Prognostic Role of Neutrophil-to-Lymphocyte Ratio in Early and Late Phase of Sepsis
}

\author{
Rajnish Kaushik, Monika Gupta1, Madhu Sharma², Debraj Jash, Neetu Jain, Nitin Sinha³, Aditya Chaudhry, Dhruva Chaudhry \\ Departments of Pulmonary and Critical Care Medicine, ${ }^{1}$ Pathology and ${ }^{2}$ Microbiology, Pt B.D. Sharma Postgraduate Institute of Medical Sciences, Rohtak, Haryana, \\ ${ }^{3}$ Department of Medicine, Dr RML Hospital, New Delhi, India
}

\section{Abstract}

Objective: Early diagnosis of sepsis is necessary to decrease morbidity and mortality. This study aims to evaluate neutrophil-to-lymphocyte ratio (NLR) as diagnostic and prognostic of early and late phase of sepsis. Methods: It was a prospective, observational study, conducted in Intensive Care and High Dependency Unit (Daycare) of the Department of Pulmonary and Critical Care Medicine (tertiary care center), Rohtak, from January 2017 to December 2017. A total of 56 cases of newly diagnosed cases of sepsis were included in the study and 20 healthy adults were taken as controls. Daily NLR was calculated in cases till the primary outcome. Results: The results suggested that NLR seems to have promising role as diagnostic and prognostic marker (with $P=0.001$ and $P=0.045$, respectively) in sepsis. Conclusion: The study suggests that NLR can be a useful diagnostic and prognostic marker in sepsis.

Keywords: Biomarkers, critical care, diagnostic, inflammatory, sepsis

\section{INTRODUCTION}

Sepsis is a life-threatening inflammatory disorder and the immune system's response to infection. In 1992, "sepsis" was formally defined as the presence of both suspected infection and two of the four criteria of the systemic inflammatory response syndrome (SIRS) ${ }^{[1]}$ Sepsis is a clinical syndrome of physiological, pathological, and biochemical abnormalities induced by infection. Mortality rates from sepsis range between $25 \%$ and $30 \%$ for severe sepsis and $40 \%$ and $70 \%$ for septic shock. ${ }^{[2]}$ A 72 -h survival rate decreases by roughly $7.7 \%$ every hour such that appropriate antimicrobial medication is delayed at the onset of infection, underscoring the need for early diagnosis techniques. ${ }^{[3]}$ Sepsis comprises early phase and late phase. Early phase is initial 5 days and after that is late phase. ${ }^{[4]}$ Diagnosis of sepsis has shifted from SIRS criteria to sepsis-3 definition involving the qSOFA (quick Sepsis Related Organ Failure Assessment). ${ }^{[5]}$

A large number of biological materials have been investigated as candidates for sepsis biomarkers. ${ }^{[6]}$ Conventionally, the white blood cell (WBC) count, erythrocyte sedimentation rate, and $\mathrm{C}$-reactive protein (CRP) are utilized as common screening laboratory tests, but these have poor sensitivity and specificity. ${ }^{[7]}$ A positive microbiological culture is considered

\begin{tabular}{|l|l|}
\hline \multicolumn{2}{|c|}{ Access this article online } \\
\hline Quick Response Code: & Website: \\
\hline & www.ijccm.org \\
\hline & \\
\hline
\end{tabular}

as gold standard for diagnosis, but this is time-consuming and sometimes leads to false-negative outcomes when the patient has previously received antibiotics. ${ }^{[8]}$

de Jager et al. investigated whether neutrophil-to-lymphocyte count ratio (NLCR) can predict bacteremia better than conventional infection markers in an emergency care unit on 92 patients. The authors found significant differences between patients with positive and negative blood cultures with respect to the CRP level, lymphocyte count, and NLCR. They concluded that in an emergency care setting, both lymphocytopenia and NLCR are better predictors of bacteremia than routine parameters such as CRP level, WBC count, and neutrophil count. ${ }^{[9]}$ Zahorec demonstrated that the ratio of neutrophil and lymphocyte counts was an easily measurable parameter for determining prognosis during Intensive Care Unit (ICU) follow-up of the patient and it

Address for correspondence: Dr. Dhruva Chaudhry, Senior Professor \& Head, Department of Pulmonary \& Critical Care, Pt B.D. Sharma Postgraduate Institute of Medical Sciences, Rohtak, Haryana, India. E-mail: dhruvachaudhry@yahoo.co.in

This is an open access journal, and articles are distributed under the terms of the Creative Commons Attribution-NonCommercial-ShareAlike 4.0 License, which allows others to remix, tweak, and build upon the work non-commercially, as long as appropriate credit is given and the new creations are licensed under the identical terms.

For reprints contact: reprints@medknow.com

How to cite this article: Kaushik R, Gupta M, Sharma M, Jash D, Jain N, Sinha N, et al. Diagnostic and prognostic role of neutrophil-to-lymphocyte ratio in early and late phase of sepsis. Indian J Crit Care Med 2018;22:660-3. 
could be used routinely in daily clinical practice. ${ }^{[10]}$ Using above all concept, this study tried to evaluate the role of ratio of neutrophils and lymphocytes as diagnostic and prognostic marker in sepsis.

\section{Methods}

\section{Study population}

It was a prospective, observational study, conducted in the Department of Pulmonary and Critical Care Medicine, Rohtak, from January 2017 to December 2017. Patients aged $>18$ years after fulfilling the criteria of newly diagnosed sepsis as per the definition of sepsis by the American College of Chest Physicians and Society of Critical Care Medicine were included in the study. ${ }^{[1]}$ Pregnant females, chronic ventilator dependents, immunocompromised participants, and patients on steroid therapy of more than 5 days were excluded from the study. Twenty normal healthy age- and sex-matched individuals of both genders were taken as controls. All the cases were followed up daily till primary outcome which was discharge, death, or shifting to step-down facility.

Absolute neutrophil counts, absolute lymphocytic count, neutrophil-to-lymphocyte ratio (NLR) were studied daily in all patients on Mindray BC-5800 five-point hematological analyzer till the primary outcome. In controls, one-time absolute neutrophil count, absolute lymphocytic count, and NLR were calculated and taken as reference value. After hematological studies, the biomedical waste generated during the procedure was discarded as per the BioMedical Waste (Management and Handling) Rules, 2011. [11]

\section{Statistical analysis}

For continuous variables, paired $t$-test was employed for having normal distribution, while for nonnormal distribution, Wilcoxon test was employed. For association of categorical variables, Chi-square test was used. For diagnostic and prognostic value, receiver operating characteristic curve (ROC) was used. Data were analyzed using IBM Corp Released 2011. IBM SPSSStatistics for Windows, Version 21.0.Armonk, NY:IBM Corp.

\section{RESULTS}

A total of 80 cases were included in the study after informed consent and subsequently 24 cases were excluded from the study. The different reasons for exclusion were pregnancy in four patients; seven were on high dose and long duration of steroid; three each were human Immunodeficiency virus positive; in late phase, already, five patients were excluded because of the unavailability of flow cytometry; one patient each had carcinoma lung and tuberculosis. Finally, 56 cases met the criteria for study [Figure 1]. Out of 56 cases, 6 expired in early phase and 50 cases were followed till late phase of sepsis. On the basis of primary outcome, participants were further divided into survivors (Group I) and nonsurvivors (Group II).
Out of 56 cases, 37 survived (Group I) and 19 cases were nonsurvivors (Group II). Median age was $30.00 \pm 28.00$ years, with $35.81 \pm 28.00$ years in Group I and $36.00 \pm 34.00$ years in Group II, but the difference was not significant [Table 1]. There were 33 males and 23 females in the study, with 15 females in Group I and 8 females in Group II. About 47\% of participants in Group II were smokers as compared to $27 \%$ in Group I. About 40.54\% of participants in Group I had comorbid illness as compared to $68.42 \%$ in Group II. Most common comorbid illness was chronic obstructive pulmonary disease present in 18 participants [Table 1]. Among the study cases, the most common site of sepsis was respiratory system (pneumonia) [Table 2].

Thirty-three (58\%) patients had septic shock at the time of enrollment, and out of which, 17 survived and 16 expired.

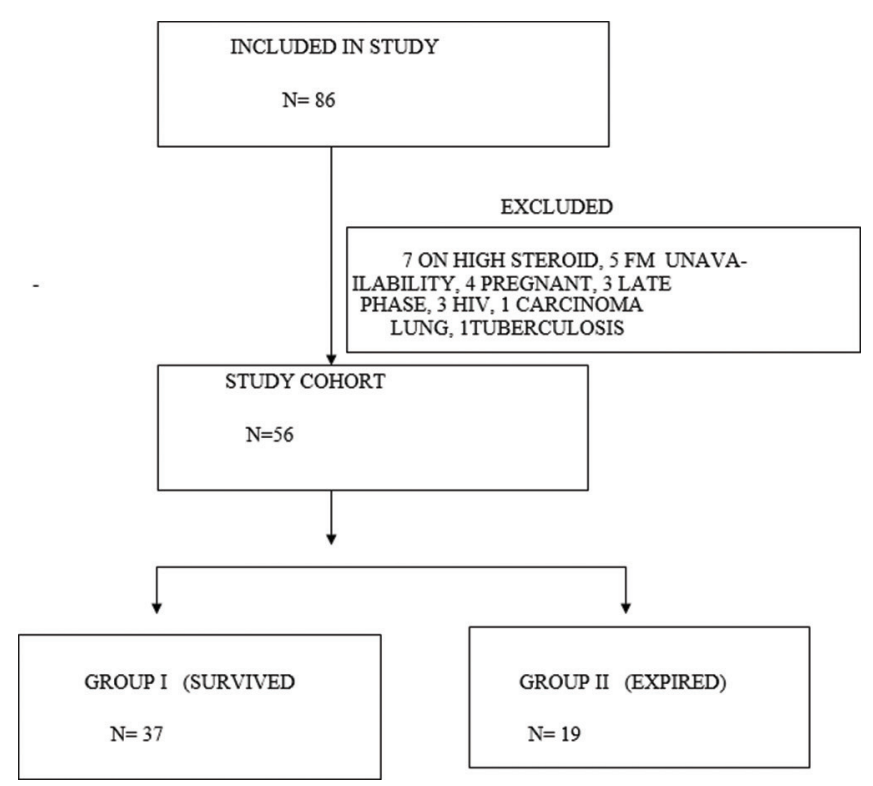

Figure 1: Study population

\begin{tabular}{lcccc}
\hline \multicolumn{5}{l}{ Table 1: Demographic profile of cases } \\
& Total cases & Group I & Group II & $\boldsymbol{P}$ \\
\hline Age (yrs) (median) & $30.00 \pm 28.0$ & $35.861 \pm 28.0$ & $36.00 \pm 34.0$ & 0.842 \\
Sex & & & & \\
$\quad$ Male & 33 & 22 & 11 & 0.918 \\
Female & 23 & 15 & 8 & \\
BMI (mean) $\left(\mathrm{kg} / \mathrm{m}^{2}\right)$ & $23.66 \pm 4.29$ & $23.33 \pm 4.4$ & $24.43 \pm 4.22$ & 0.625 \\
Smoking & $19(56)$ & $10(37)$ & $9(19)$ & 0.128 \\
Residence & & & & \\
$\quad$ Urban & 34 & 23 & 11 & 0.757 \\
$\quad$ Rural & 22 & 14 & 8 & \\
Comorbidity & & & & \\
Diabetes & 6 & 4 & 2 & \\
Hypertension & 4 & 2 & 2 & \\
CAD & 3 & 1 & 2 & \\
COPD & 9 & 5 & 4 & \\
Old TB & 6 & 3 & 3 & \\
\hline
\end{tabular}


Mean APACHE II score was significantly higher in Group II $(P<0.001)$ [Table 2].

\section{Control group characteristics}

Mean age of persons in control group was $35 \pm 13.00$ years. There were more males (17) than females (3) [Table 3a]. Mean total lymphocyte count was $6535.75 \pm 1665.11 / \mathrm{mm}^{3}$. Median NLR was $2.0 \pm 0.94$.

\section{Neutrophil-to-lymphocyte ratio among cases and controls} In sepsis cases, NLR of early phase of sepsis was significantly higher as compared to controls. ROC for NLR on day 1 (NLR1) showed area under the ROC curve (AUC) of 0.911 with significance of $P<0.001$ with sensitivity of $87.5 \%$ and specificity of $90 \%$ at a value of $\geq 3.3$ [Figure 2 and Table 3b]. Among Group I and Group II of cases, NLR1 was not significantly different, but late phase NLR on day 5 (NLR5) was significantly low in survivors (Group I) [Table 3c]. For NLR5, it had AUC of 0.732 with significance of $P<0.045$ with sensitivity of $73 \%$ and specificity of $71 \%$ at a value of 8.3 [Figure 3].

\section{Discussion}

Early diagnosis of sepsis is essential to decrease the morbidity and mortality. Microbiological culture has been considered as gold standard for diagnosis of sepsis, but it is time-consuming, and yield is poor due to previously received antibiotics and falsely positive secondary to contamination during inoculation techniques. Due to limitations of culture, there has been constant effort to discover new effective markers of sepsis with good diagnostic and prognostic yields. In the present study, level of NLR is found to be elevated significantly in subjects as compared to controls. The role of NLR was studied in sepsis by Jilma et al., and they found persistently high levels of neutrophil count $(300 \%)$ in patients of sepsis

\section{Table 2: Clinical profile of cases}

\begin{tabular}{|c|c|c|c|c|}
\hline & Total cases & Group I & Group II & $P$ \\
\hline \multicolumn{5}{|l|}{ Source of infection } \\
\hline Respiratory & 40 & 28 & 12 & \\
\hline Urinary/renal & 8 & 4 & 4 & \\
\hline Abdominal & 4 & 2 & 2 & \\
\hline Cardiac & 1 & 0 & 1 & \\
\hline Neurological & 3 & 3 & 0 & \\
\hline Septic shock & 33 & $17(45 \%)$ & $16(84 \%)$ & 0.010 \\
\hline Days in ICU (median) & $4.0 \pm 6.0$ & $10.00 \pm 4.80$ & $6.0 \pm 7.0$ & 0.044 \\
\hline Days on mechanical ventilation (median) & $5.50 \pm 50$ & $5.50 \pm 3.00$ & $5.5 \pm 6.0$ & 0.707 \\
\hline APACHE II (mean) & $26.79 \pm 4.1$ & $24.19 \pm 5.0$ & $29.26 \pm 3.95$ & $<0.001$ \\
\hline
\end{tabular}

\section{Table 3a: Control characteristics}

\begin{tabular}{lccccc}
\hline Group & Age (median) (Yrs) & Sex (median) & HB (median) (mg/dl) & TLC (mean) (/mm $\left.{ }^{3}\right)$ & NLR1 (median) $^{(m .16}$ \\
\hline Control & $35.00 \pm 13.00$ & $3 \mathrm{~F} / 17 \mathrm{M}$ & $14.15 \pm 1.4$ & $6535.75 \pm 1665.11$ & $2.0 \pm 0.94$ \\
\hline
\end{tabular}

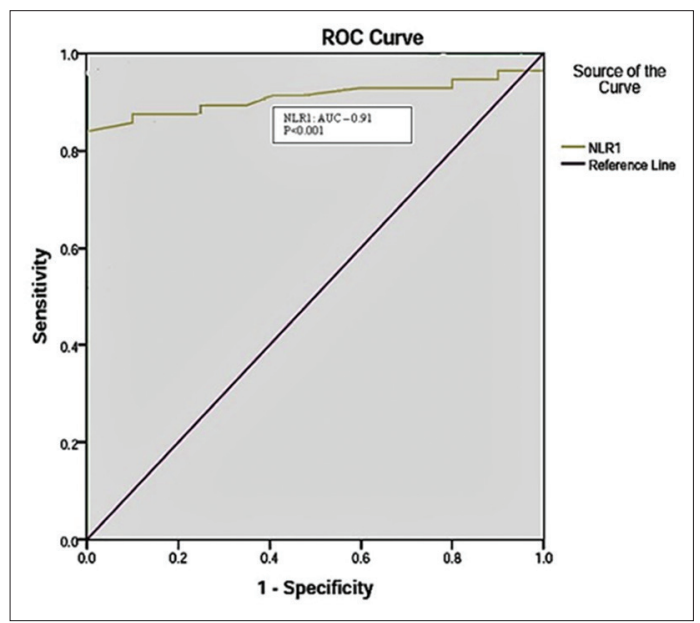

Figure 2: Receiver operating characteristic curve of neutrophil-to-lymphocyte ratio on day 1 for diagnostic value

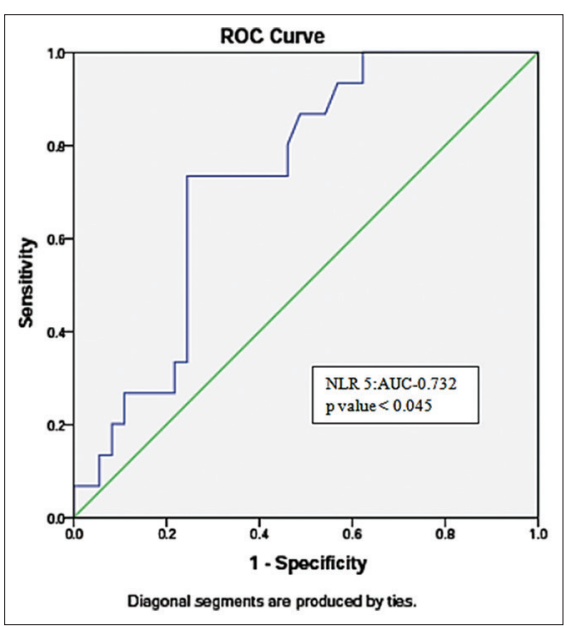

Figure 3: Receiver operating characteristic curve of late phase neutrophil-to-lymphocyte ratio on day 5 for prognostic value 
Table 3b: Neutrophil-to-lymphocyte ratio Day 1 among cases and controls

\begin{tabular}{cccc}
\hline & Cases & Controls & Significance \\
\hline NLRI & $8.5 \pm 5.95$ & $2.0 \pm 0.94$ & $<0.001$ \\
\hline
\end{tabular}

Table 3c: Neutrophil-to-lymphocyte ratio Day 1 and neutrophil-to-lymphocyte ratio Day 5 among Group I and Group II

\begin{tabular}{lcccc}
\hline & Total cases & Group I & Group II & $\boldsymbol{P}$ \\
\hline NLR1 (median) & $8.50 \pm 5.95$ & $8.45 \pm 5.63$ & $8.60 \pm 6.20$ & 0.483 \\
NLR5 (median) & $5.90 \pm 5.60$ & $4.90 \pm 6.38$ & $8.60 \pm 5.70$ & 0.009 \\
\hline
\end{tabular}

and they concluded that persistent lymphocytopenia was associated with poor outcome in sepsis. ${ }^{[12]}$ de Jager et al. observed NLR as a better predictor of severity and outcome in bacteremia than conventional markers. ${ }^{[9]}$ Zahorec discovered NLR to be an easily measurable and having better correlation with sepsis severity and 28-day mortality than neutrophilia, lymphocytopenia, and severity of clinical course. ${ }^{[10]}$

We found NLR to be significantly higher in cases with high-neutrophil and low-lymphocyte count as compared to controls on day 1 which is in coherence with the above studies..$^{[9,10,12]}$ The sensitivity and specificity of NLR as a diagnostic marker was $87.5 \%$ and $90 \%$, respectively. Persistent lymphocytopenia was also found to be associated with poor outcome as demonstrated by Jilma et al. ${ }^{[12]}$ In Group II, NLR was found to be persistently high and in Group I NLR came down significantly in the late phase signifying that decrease in NLR was associated with survival. High NLR5, i.e., at the end of early phase was significantly associated with poor outcome with a prognostic value having $73 \%$ sensitivity and $71 \%$ specificity. Participants who had high value of NLR5 had poor outcome with high APACHE II score and longer ICU stay. Participants with high NLR5 correlated positively with severity of disease and mortality as also shown by Zahorec. ${ }^{[10]}$

Like any study, our study also has few limitations primarily of small sample size. Second, culture positivity rate was only $25 \%$; being a tertiary care institute, most of the patients in our study were referred and had already received antimicrobials.

\section{Conclusion}

Elevated levels of NLR are seen in early phase of sepsis and thus helpful in making a diagnosis, especially when obtaining microbiological culture poses limitation in terms of time and low-positive rate. Late phase value of this inflammatory biomarker is also helpful in dictating the prognosis. In addition to the prognostic value, this marker can also be exploited to discontinue antimicrobials as the patient improves. However, further research is desired with large sample size and in patients of noninfective inflammatory conditions to strengthen the role of this potential marker in sepsis.

\section{Financial support and sponsorship}

Nil.

\section{Conflicts of interest}

There are no conflicts of interest.

\section{RefEREnCES}

1. American College of Chest Physicians/Society of Critical Care Medicine Consensus Conference: Definitions for sepsis and organ failure and guidelines for the use of innovative therapies in sepsis. Crit Care Med 1992;20:864-74.

2. Lever A, Mackenzie I. Sepsis: Definition, epidemiology, and diagnosis. BMJ 2007;335:879-83.

3. Kumar A, Roberts D, Wood KE, Light B, Parrillo JE, Sharma S, et al. Duration of hypotension before initiation of effective antimicrobial therapy is the critical determinant of survival in human septic shock. Crit Care Med 2006;34:1589-96.

4. Riché F, Gayat E, Barthélémy R, Le Dorze M, Matéo J, Payen D, et al. Reversal of neutrophil-to-lymphocyte count ratio in early versus late death from septic shock. Crit Care 2015;19:439.

5. De Backer D, Dorman T. Surviving sepsis guidelines: A continuous move toward better care of patients with sepsis. JAMA 2017;317:807-8.

6. Siqueira-Batista R, Gomes AP, Calixto-Lima L, Vitorino RR, Perez MC, Mendonça EG, et al. Sepsis: An update. Rev Bras Ter Intensiva 2011;23:207-16.

7. Davis BH. Improved diagnostic approaches to infection/sepsis detection. Expert Rev Mol Diagn 2005;5:193-207.

8. Peters RP, van Agtmael MA, Danner SA, Savelkoul PH, Vandenbroucke-Grauls CM. New developments in the diagnosis of bloodstream infections. Lancet Infect Dis 2004;4:751-60.

9. de Jager CP, van Wijk PT, Mathoera RB, de Jongh-Leuvenink J, van der Poll T, Wever PC, et al. Lymphocytopenia and neutrophil-lymphocyte count ratio predict bacteremia better than conventional infection markers in an emergency care unit. Crit Care 2010;14:R192.

10. Zahorec R. Ratio of neutrophil to lymphocyte counts - Rapid and simple parameter of systemic inflammation and stress in critically ill. Bratisl Lek Listy 2001;102:5-14.

11. Draft Biomedical Waste (Management and Handling) Rules. New Delhi: Government of India, Ministry of Environment and Forests; 2011. Available from: http://www.moef.nic.in/downloads/public-information/ salient-features-draft-bmwmh.pdf. [Last accessed on 2011 Jun 21].

12. Jilma B, Blann A, Pernerstorfer T, Stohlawetz P, Eichler HG, Vondrovec B, et al. Regulation of adhesion molecules during human endotoxemia. No acute effects of aspirin. Am J Respir Crit Care Med 1999;159:857-63. 